\title{
Coupling of Chiralities in Spin and Physical Spaces: The Möbius Ring as a Case Study
}

\author{
Oleksandr V. Pylypovskyi, ${ }^{1}$ Volodymyr P. Kravchuk, ${ }^{2, *}$ Denis D. Sheka, ${ }^{1}$ Denys Makarov, ${ }^{3}$ \\ Oliver G. Schmidt, ${ }^{3}$ and Yuri Gaididei ${ }^{2}$ \\ ${ }^{1}$ Taras Shevchenko National University of Kiev, 01601 Kiev, Ukraine \\ ${ }^{2}$ Bogolyubov Institute for Theoretical Physics, 03143 Kiev, Ukraine \\ ${ }^{3}$ Institute for Integrative Nanosciences, IFW Dresden, 01069 Dresden, Germany
}

(Received 20 October 2014; revised manuscript received 30 March 2015; published 15 May 2015)

\begin{abstract}
We show that the interaction of the magnetic subsystem of a curved magnet with the magnet curvature results in the coupling of a topologically nontrivial magnetization pattern and topology of the object. The mechanism of this coupling is explored and illustrated by an example of a ferromagnetic Möbius ring, where a topologically induced domain wall appears as a ground state in the case of strong easy-normal anisotropy. For the Möbius geometry, the curvilinear form of the exchange interaction produces an additional effective Dzyaloshinskii-like term which leads to the coupling of the magnetochirality of the domain wall and chirality of the Möbius ring. Two types of domain walls are found, transversal and longitudinal, which are oriented across and along the Möbius ring, respectively. In both cases, the effect of magnetochirality symmetry breaking is established. The dependence of the ground state of the Möbius ring on its geometrical parameters and on the value of the easy-normal anisotropy is explored numerically.

DOI: 10.1103/PhysRevLett.114.197204

PACS numbers: 75.75.-c, 75.10.Hk, 75.30.Et
\end{abstract}

Curvature-driven modification of physical properties of systems with nontrivial geometry is the subject of intensive research in various areas of physics [1,2], e.g., electronic properties of graphene [3], molecular alignment in liquid crystals [4], physics of superconductors [5,6], and macromolecular structures [7]. In physics of nanomagnetism, it is possible to distinguish two groups of curvature-induced effects, namely, magnetochiral effects [8-10] and topologically induced magnetization patterning [10,11]. The first family unites the phenomena of curvature-induced chiral symmetry breaking [12]. The latter typically originates from the Dzyaloshinskii-like term appearing in the curvilinear form of the exchange interaction $[13,14]$. Effects of the second group appear in curvilinear magnets, where orientation of the anisotropy axis is determined by the geometry, e.g., along the normal direction. Thereby, magnetic vortices appear in the ground state of the spherical magnetic shell with easy-surface anisotropy [10], domain walls appear in the ground state of a Möbius ring with easynormal anisotropy [11].

Up to now, these two families of effects were considered to be independent. Here, we demonstrate that geometry of the Möbius ring unites these two families of curvature effects; namely, the topologically induced magnetization patterns experience the chirality symmetry breaking. The Möbius ring is a nonorientable surface, hence, its topology forces a discontinuity of any normal vector field. This is the

Published by the American Physical Society under the terms of the Creative Commons Attribution 3.0 License. Further distribution of this work must maintain attribution to the author(s) and the published article's title, journal citation, and DOI. origin of the domain structure formation for the Möbius ring with strong easy-normal anisotropy, similar to the nucleation of disclination lines in chiral nematics [15]. The domain wall for the Möbius geometry was obtained numerically [11] within the model of a classical Heisenberg ferromagnet. Constrained by the topology, we name such a magnetization structure as a topologically induced domain wall. We demonstrate analytically that the topological properties of a domain wall on a Möbius ring depend on the topological properties of the underlying surface, namely, the magnetochirality of the domain wall is determined by the chirality of the Möbius ring. Additionally, using the full scale micromagnetic simulations with magnetostatic interaction taken into account, we confirm these results and also build a ground states diagram for Möbius rings.

First, we formalize the notion of the Möbius ring of finite thickness. The two-dimensional Möbius ring can be parametrized in the form $\boldsymbol{s}(\chi, \xi)=x(\chi, \xi) \hat{\boldsymbol{x}}+y(\chi, \xi) \hat{\boldsymbol{y}}+$ $z(\chi, \xi) \hat{z}$ with

$$
\begin{aligned}
x+i y & =\left(R+\xi \cos \frac{\chi}{2}\right) e^{i \chi}, \quad 0 \leq \chi<2 \pi ; \\
z & =\mathcal{C} \xi \sin \frac{\chi}{2}, \quad-\frac{w}{2} \leq \xi \leq \frac{w}{2} ;
\end{aligned}
$$

where $R$ and $w$ denote the radius and width of the ring, respectively, see Fig. 1 . The curvilinear coordinates $\chi$ and $\xi$ correspond to the azimuthal angle and position along the ring width, respectively. The Möbius ring chirality $\mathcal{C}= \pm 1$ determines orientation of the ring twist, when moving along the azimuthal direction, namely, counterclockwise $(\mathcal{C}=+1)$ or clockwise $(\mathcal{C}=-1)$. 


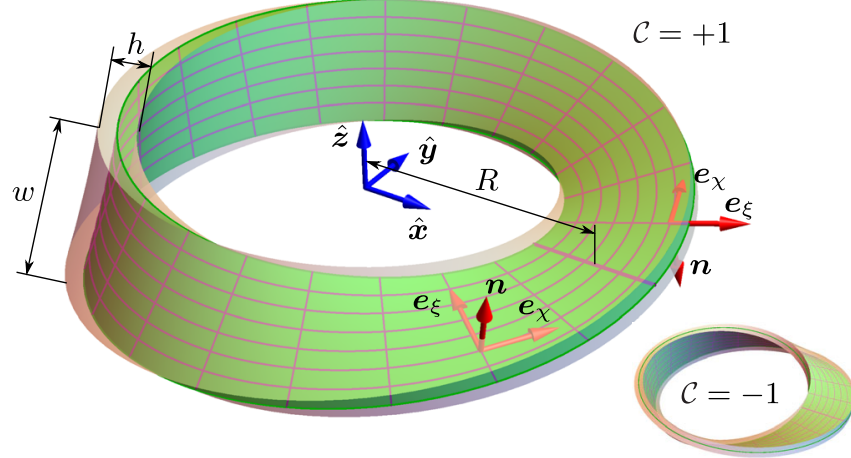

FIG. 1 (color online). Geometrical notations of the problem: the 2D Möbius ring with radius $R$ and width $w$ is defined parametrically by (1) and the corresponding 3D Möbius ring of finite thickness $h$ is determined by (2). Möbius rings with opposite chiralities $\mathcal{C}$ are shown.

Using (1), we introduce the orthonormal curvilinear basis as $\boldsymbol{e}_{\alpha}=\boldsymbol{g}_{\alpha} /\left|\boldsymbol{g}_{\alpha}\right|$, where $\boldsymbol{g}_{\alpha}=\partial_{\alpha} \boldsymbol{\mathcal { S }}$ and $\alpha \in\{\chi, \xi\}$. The vector of the normal is $\boldsymbol{n}=\boldsymbol{e}_{\chi} \times \boldsymbol{e}_{\xi}$, see Fig. 1 .

As a result, the three-dimensional Möbius ring of finite thickness $h$ is now defined as the following space domain:

$$
\boldsymbol{r}(\chi, \xi, \eta)=\boldsymbol{s}(\chi, \xi)+\eta \boldsymbol{n}, \quad-\frac{h}{2} \leq \eta \leq \frac{h}{2} ;
$$

where the third curvilinear coordinate $\eta$ determines the position along the ring thickness. The definition (2) is valid for the case of small thickness: $h \ll R-(w / 2)$.

To describe the space distribution of the unit magnetization vector $\boldsymbol{m}(\boldsymbol{r})$, it is convenient to introduce the angular parametrization associated with the local curvilinear basis

$$
\boldsymbol{m}=(\sin \theta \cos \phi) \boldsymbol{e}_{\chi}+(\sin \theta \sin \phi) \boldsymbol{e}_{\xi}+(\cos \theta) \boldsymbol{n},
$$

with the magnetization angles $\theta=\theta(\chi, \xi)$ and $\phi=\phi(\chi, \xi)$.

We start with the case of strong easy-normal anisotropy, when the formation of the topologically induced domain walls is expected [11]. Two types of domain walls are found using full scale micromagnetic simulations, namely, transversal $(t)$ and longitudinal $(\ell)$, see Fig. 2(c) and Fig. 2(e), respectively. We analyze the properties of the topologically induced domain walls under two assumptions: (i) the magnetostatic contribution is negligibly small as compared with the anisotropy contribution, (ii) the ring thickness $h$ is small enough to ensure the magnetization uniformity along the normal direction $\boldsymbol{n}$. Thus, the total energy of the system reads $E=h \int\left(\mathcal{E}_{\text {ex }}+\mathcal{E}_{\text {an }}\right) d \mathcal{S}$, where $d \mathcal{S}$ is the curvilinear element of the surface area. Here, $\mathcal{E}_{\mathrm{ex}}=A \sum_{i=x, y, z}\left(\boldsymbol{\nabla} m_{i}\right)^{2}$ is the exchange contribution with $A$ being the exchange constant, and $\mathcal{E}_{\text {an }}=-K(\boldsymbol{m} \cdot \boldsymbol{n})^{2}$ is the easy-normal anisotropy density with $K>0$ being the anisotropy constant. In terms of the angular variables (3), the anisotropy term reads as

$$
\mathcal{E}_{\text {an }}=-K \cos ^{2} \theta
$$

The exchange energy density for an arbitrary curvilinear thin ferromagnetic film can be presented in the form [13]

$$
\frac{\mathcal{E}_{\mathrm{ex}}}{A}=[\boldsymbol{\nabla} \theta-\boldsymbol{\Gamma}(\phi)]^{2}+\left[\sin \theta(\boldsymbol{\nabla} \phi-\boldsymbol{\Omega})-\cos \theta \frac{\partial \boldsymbol{\Gamma}(\phi)}{\partial \phi}\right]^{2} .
$$

(a)

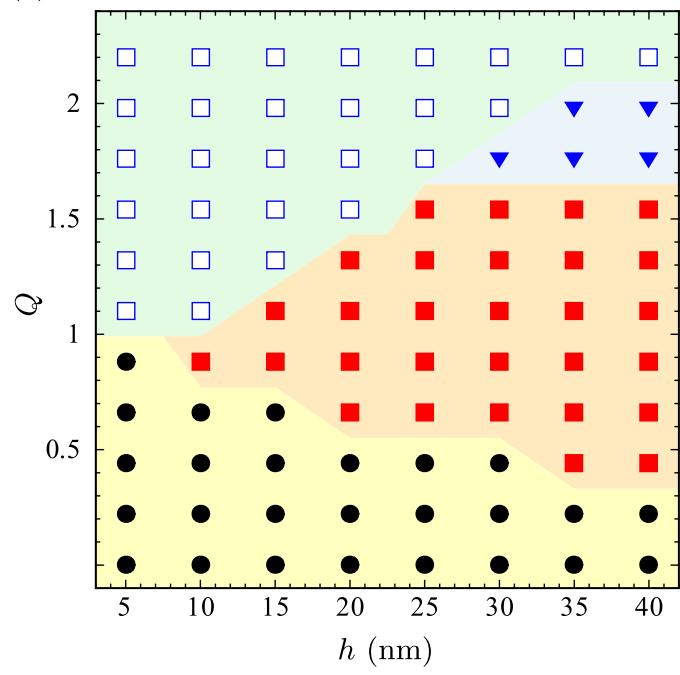

(b)

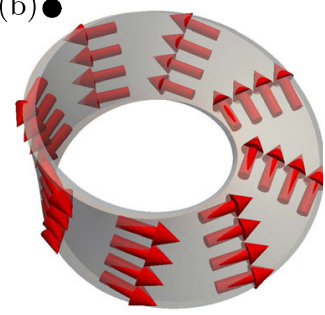

(d)

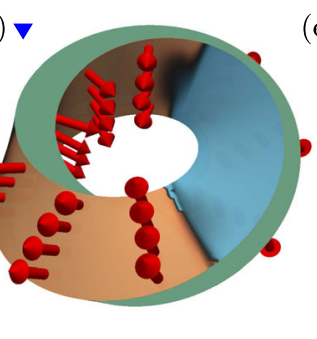

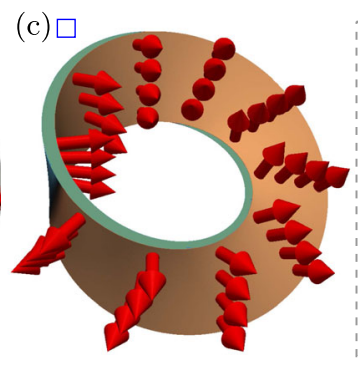

(e)

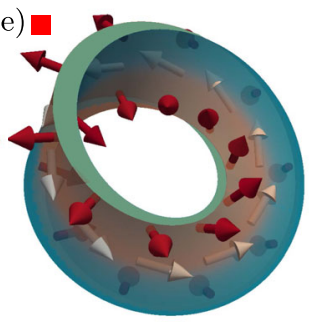

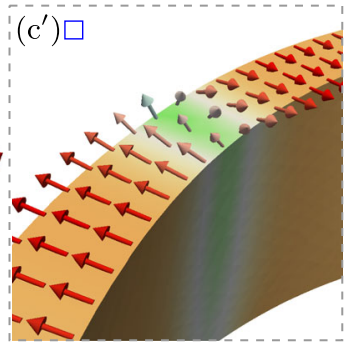

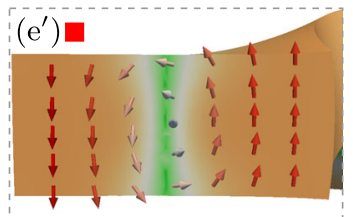

FIG. 2 (color online). Diagram of ground states of magnetic Möbius rings with fixed radius $R=100 \mathrm{~nm}$ and width $w=80$, see (a). The magnetization distributions of possible ground states are shown in the middle part in a large scale: (b) vortex state (marked by disks on the diagram), (c) state with single transversal Bloch domain wall (open squares), (d) state with three transversal Bloch walls (filled triangles), (e) states with longitudinal domain wall (filled squares). The detailed structures of the transverse and longitudinal domain walls are shown in $\left(\mathrm{c}^{\prime}\right)$ and $\left(\mathrm{e}^{\prime}\right)$, respectively. 
Here, the del operator is used in its curvilinear form $\boldsymbol{\nabla} \equiv\left(g_{\alpha \alpha}\right)^{-1 / 2} \boldsymbol{e}_{\alpha} \partial_{\alpha}$, where $g_{\alpha \beta}=\boldsymbol{g}_{\alpha} \cdot \boldsymbol{g}_{\beta}$ are elements of the metric tensor, and the Einstein summation rule is applied here and everywhere below. Vector $\boldsymbol{\Gamma}=\Gamma_{\alpha} \boldsymbol{e}_{\alpha}$ is tangential to the surface, and it is defined as

$$
\boldsymbol{\Gamma}(\phi)=\left\|H_{\alpha \beta}\right\| \cdot\left\|\begin{array}{c}
\cos \phi \\
\sin \phi
\end{array}\right\|, \quad\left\|H_{\alpha \beta}\right\|=\left\|\frac{b_{\alpha \beta}}{\sqrt{g_{\alpha \alpha} g_{\beta \beta}}}\right\|,
$$

where $b_{\alpha \beta}=\boldsymbol{n} \cdot \partial_{\beta} \boldsymbol{g}_{\alpha}$ are elements of the second fundamental form and the vector $\boldsymbol{\Omega}$ represents the modified spin connection $\boldsymbol{\Omega}=\left(g_{\alpha \alpha}\right)^{-1 / 2}\left(\boldsymbol{e}_{\chi} \cdot \partial_{\alpha} \boldsymbol{e}_{\xi}\right) \boldsymbol{e}_{\alpha}$.

Basic properties of the $t$ wall can be analyzed using the ansatz

$$
\theta^{t}=2 \arctan e^{p(\chi-X) / \sigma}, \quad \phi^{t}=\mathfrak{c} p \frac{\pi}{2},
$$

which describes the structure of a typical Bloch domain wall [16] aligned across the Möbius ring [17]. It contains two variational parameters, namely, azimuthal angle $X$ which determines the position of the $t$ wall and its angular width $\sigma$. The quantity $p= \pm 1$ determines the wall of kink $(p=+1)$ or antikink $(p=-1)$ type. The magnetochirality $\mathfrak{C}= \pm 1$ determines the direction of the magnetization reorientation within the domain wall, when moving along the azimuthal direction: counterclockwise $(\mathfrak{E}=+1)$ or clockwise $(\mathfrak{C}=-1)$. The usage of the ansatz (6) for a Möbius ring has a restriction $\sigma \ll X \ll 2 \pi-\sigma$.

Now, we substitute ansatz (6) into (4) and (5) and perform the integration over the curvilinear surface area with the area element $d \mathcal{S}=\sqrt{\operatorname{det}\left(g_{\alpha \beta}\right)} d \chi d \xi$. Using the natural condition $\sigma \ll 1$ and applying the narrow ring approximation $w / R \ll 1$, one can write the total energy of the $t$ wall as follows:

$\frac{E^{t}}{2 A h} \approx \frac{w}{R}\left[\frac{1}{\sigma}+\mathfrak{C C} \frac{\pi}{2}+\sigma\left(\cos X-\frac{1}{4}\right)\right]+\sigma \frac{R w}{\delta^{2}}+$ const,

where $\delta=\sqrt{A / K}$, and the constant terms do not depend on the variational parameters and on the chiralities.

Remarkably, according to (7), a coupling between the chirality of the Möbius ring $\mathcal{C}$ and the magnetochirality of the $t$ wall $\mathfrak{C}$ takes place. The $t$ walls with opposite chiralities possess different energies with $\Delta E^{t}=2 \pi A h w / R$ (the $t$ wall with chirality $\mathfrak{C}=-\mathcal{C}$ has lower energy). We note that the energy (7) is independent of $p$.

The energy (7) reaches its minimum at the following values of the variational parameters $X_{0}=\pi, \sigma_{0} \approx \delta / R$. In accordance with the parametrization (1), the equilibrium position of the domain wall $X_{0}=\pi$ corresponds to the "vertical" place on the Möbius ring, see Figs. 1 and 2(c).
The law $E^{t} \propto \cos X$ is well reproduced by full scale micromagnetic simulations [18].

To consider the $\ell$ wall, see Fig. 2(e), we use the analogous ansatz

$$
\theta^{\ell}=2 \arctan e^{p(\xi / d)}, \quad \phi^{\ell}=\pi \frac{\mathfrak{C}+p}{2},
$$

which describes a Bloch domain wall aligned along the Möbius ring. It should be noted that the $\ell$ wall cannot be of Neel type due to topological reasons. The domain wall width $d$ is a variational parameter. Using the condition $d \ll w \ll R$, we obtain the following expression for the total energy of the $\ell$ wall state:

$$
\frac{E^{\ell}}{4 \pi A h} \approx \frac{R}{d}-\frac{\pi}{2} \mathcal{C}\left(5+c_{0} \frac{d}{R}+\frac{d R}{\delta^{2}}+\right.\text { const, }
$$

where $c_{0}=1 / 4+\pi^{2} / 96 \approx 0.353$. Accordingly to (9), the chiralities coupling appears for the $\ell$ wall as well as for the case of the $t$ wall. The $\ell$ walls with opposite chiralities are separated by the energy gap $\Delta E^{\ell}=4 \pi^{2} A h$ (the domain wall with $\mathfrak{C}=\mathcal{C}$ has lower energy). The equilibrium value of the domain wall width is $d \approx \delta$.

For both domain wall types, the effect of the magnetochirality symmetry breaking originates from the effective Dzyaloshinskii-like term $\mathcal{E}_{\mathrm{ex}}^{D}=-2 A(\boldsymbol{\Gamma} \cdot \boldsymbol{\nabla} \theta)$ in the curvilinear form of the exchange energy (5). This is consistent with the phenomenon of domain wall chiral symmetry breaking due to Dzyaloshinskii-Moriya interaction in planar systems [19].

It should be noted that the chirality coupling terms in (7) and (9) originate from the strip twisting, hence, they are not unique for the Möbius geometry and will appear even when considering a straight strip with a twist (local or uniformly distributed). We choose the Möbius geometry because, in this case, two curvature effects are united, namely, the chirality symmetry breaking appears for a topologically induced pattern.

The curvature-induced magnetochirality effects are already known for domain structure (twisting of domains) [8], spin waves (asymmetry in the spin waves propagation in nanotubes) [12]. Its analogy with the DzyaloshinskiiMoriya interaction was discussed in Ref. [12]. The presented approach highlights the origin of these magnetochiral effects.

According to (7) and (9), the total energy of a $t$ wall is always lower than the total energy of a $\ell$ wall. However, the carried out analysis is valid for the case of very strong anisotropy, when the magnetostatic interaction can be neglected. To consider cases when the magnetostatic contribution is comparable or greater then the anisotropy interaction, we perform the full scale micromagnetic simulations with three magnetic interactions taken into account. Thus, the total energy density reads $\mathcal{E}=\mathcal{E}_{\text {ex }}+\mathcal{E}_{\text {an }}+\mathcal{E}_{d}$. Here, $\mathcal{E}_{d}=-M_{s}\left(\boldsymbol{H}_{d} \cdot \boldsymbol{m}\right) / 2$ is the magnetostatic energy 
density, with $M_{s}$ being the saturation magnetization and $\boldsymbol{H}_{d}$ being the stray field. To clarify the role of the magnetostatic contribution, we study the change of the ground state of the system under variation of two parameters, namely, thickness $h$ and a quality factor $Q=K /\left(2 \pi M_{s}^{2}\right)$, the latter relates the anisotropy to the magnetostatic contribution [20].

For our study, we fix the ring radius to $R=100 \mathrm{~nm}$ and width to $w=80 \mathrm{~nm}$. The calculations are performed for a magnetic material with $A=1.3 \times 10^{-11} \mathrm{~J} / \mathrm{m}, M_{s}=8.6 \times$ $10^{5} \mathrm{~A} / \mathrm{m}$. Additionally, we introduce the easy-normal anisotropy with constant $K$ varying [21] in the way that $Q \in[0,2.2]$. The thickness is varied [22] within the range $h \in[5,40] \mathrm{nm}$. For a certain set of parameters, we use the MAGPAR code [23] to numerically minimize the total magnetic energy of the system $E=\int \mathcal{E} d^{3} \boldsymbol{r}$. To obtain the ground state among the variety of possible metastable states, we applied a large number of various initial states [24] for the minimization procedure in each case. The results are presented in Fig. 2.

We conclude, from Fig. 2(a), that the ground state of the system with low anisotropy is a vortex magnetization distribution, with the magnetization vectors tangentially aligned to the Möbius surface, see Fig. 2(b). This is the typical situation for magnetically soft nanomagnets with symmetric shape, where the vortex state dominates as a result of competition between exchange and magnetostatic interactions only, e.g., in magnetic nanodisks [25] and nanorings [26]. The vortex states with magnetochiralities of opposite signs are energetically equivalent for the Möbius ring.

In the opposite case of high easy-normal anisotropy, the $t$ wall appears as the ground state, see Figs. 2(c) and 2(c'). According to Fig. 2( $\left.\mathrm{c}^{\prime}\right)$, the $t$ wall magnetochirality $\mathfrak{C}=-1$ is opposite to the Möbius ring chirality $\mathcal{C}=+1$. This confirms the conclusion about the coupling of chiralities of the object and magnetization pattern, which follows from (7). Moreover, change of the Möbius ring chirality to the opposite one leads to the corresponding switching of the magnetochirality of the $t$ wall [18].

We found that the number of $t$ walls increases with increasing thickness. For instance, states with three $t$ walls are found, see Fig. 2(d). We find out that only an odd number of $t$ walls is possible. The appearance of a multidomain structure is the typical consequence of magnetostatic interaction, because the creation of domains leads to stray field energy minimization.

For the Möbius rings of small thickness, the transition between the vortex state and $t$ wall state appears for $Q \approx 1$. However, for larger thicknesses there is a range of $Q$, where the state with the $\ell$ wall is the ground state, see Figs. 2(a) and 2(e). According to Fig. 2(e), the $\ell$ wall magnetochirality $\mathfrak{C}=+1$ coincides with the Möbius ring chirality $\mathcal{C}=+1$. This supports the conclusion about the objectpattern chirality coupling, which follows from (9). Similar to the case with the $t$ wall, the alternation of the Möbius ring chirality to the opposite one leads to the corresponding flip of the magnetochirality of the $\ell$ wall [27]. The $\ell$ wall is an asymmetric Bloch domain wall [28], see Fig. 2(e'). The influence of magnetostatics leads to an asymmetric deformation of the Bloch domain wall such that, for thick samples, the vortex formation along the wall is obtained [28], see Fig. 2(e').

The achieved effect of the coupling of chiralities of the vector (director) field subsystem and the curvilinear substrate is a general one for systems with an exchange-like interaction. For instance, it is expected for nematics in one constant approximation [29]. As it was recently demonstrated [15], the immersing of the Möbius shaped particle in the liquid crystal induces linear topological defects in the director field of the nematic. The induced defect has the form of a closed disclination loop encircling the Möbius strip [15]. The sign of strength of this loop is opposite inside and outside the Möbius strip, and therefore, the loop can be characterized by the chirality. We expect the coupling of chiralities of the disclination loop and the Möbius strip analogously to the chirality coupling in the magnetic system described above.

The presence of the topologically protected domain wall in the Möbius strip out of out-of-plane magnetized material can be used to realize nonvolatile magnetic storage media. As was demonstrated above, the $t$ wall state is doubly degenerated with respect to the binary quantity $p= \pm 1$. For a domain wall on an orientable surface, the quantity $p$ plays the role of the topological charge which is conserved. However, alternation of $p$ is not forbidden in the Möbius geometry, e.g., by application of the pulse of the magnetic field along $z$ axis (if the domain wall is situated in the "vertical" part of the Möbius strip). Furthermore, the possibility of controlling the chirality of the domain walls by local twists or curvatures could be of potential interest for the racetrack memory devices [30]. For the latter, it was recently established that the chirality of domain walls in magnetic nanostrips determines their mobility and propagation direction [31,32]. It should also be noted that, for the case of strong magnetoelastic coupling, one can expect a "feedback" effect: the chiral magnetization structure can induce a mechanical twisting of a flexible stripe. This can be especially important for applications in flexible and stretchable magnetoelectronic devices [33].

In summary, we demonstrate that the combined curvature-induced effect appears for the Möbius shaped magnetic nanoring with easy-normal anisotropy, namely, the chirality symmetry breaking takes place for both types of the topologically induced domain walls, transversal and longitudinal. This effect is driven by the effective Dzyaloshinskii-like term, which originates from the curvilinear form of the exchange interaction. It is also shown that the "vertical" part of the ring is the equilibrium position of the transverse domain wall.

Experimental verification of the predicted effects is foreseeable as a micrometer sized single crystal Möbius 
ring is already realized experimentally [34]. The experimental realization can be addressed to all-electrical measurements by monitoring the quantum spin Hall effect [35] or topological Hall effect [36] in the Möbius ring.

This work completes the broad theoretical studies of various physical phenomena for the Möbius geometry [37-46] by including magnetic phenomena.

We thank Professor Vladimir M. Fomin (IFW Dresden) for inspiring discussions. This work is financed in part via the ERC within the EU Seventh Framework Programme (ERC Grant No. 306277) and the EU FET Programme (FET-Open Grant No. 618083). O. V. P. acknowledgements the support from DAAD (Code No. 91530902-FSK).

*vkravchuk@bitp.kiev.ua

[1] M. J. Bowick and L. Giomi, Adv. Phys. 58, 449 (2009).

[2] A. M. Turner, V. Vitelli, and D. R. Nelson, Rev. Mod. Phys. 82, 1301 (2010).

[3] F. de Juan, A. Cortijo, M. A. H. Vozmediano, and A. Cano, Nat. Phys. 7, 810 (2011).

[4] G. Napoli and L. Vergori, Phys. Rev. Lett. 108, 207803 (2012)

[5] V. Vitelli and A. M. Turner, Phys. Rev. Lett. 93, 215301 (2004).

[6] V. M. Fomin, R. O. Rezaev, and O. G. Schmidt, Nano Lett. 12, 1282 (2012).

[7] R. S. Forgan, J.-P. Sauvage, and J. F. Stoddart, Chem. Rev. 111, 5434 (2011).

[8] C. Dietrich, R. Hertel, M. Huber, D. Weiss, R. Schäfer, and J. Zweck, Phys. Rev. B 77, 174427 (2008).

[9] M. Yan, C. Andreas, A. Kakay, F. Garcia-Sanchez, and R. Hertel, Appl. Phys. Lett. 100, 252401 (2012).

[10] V. P. Kravchuk, D. D. Sheka, R. Streubel, D. Makarov, O. G. Schmidt, and Y. Gaididei, Phys. Rev. B 85, 144433 (2012).

[11] M. Yoneya, K. Kuboki, and M. Hayashi, Phys. Rev. B 78, 064419 (2008).

[12] R. Hertel, SPIN 03, 1340009 (2013).

[13] Y. Gaididei, V. P. Kravchuk, and D. D. Sheka, Phys. Rev. Lett. 112, 257203 (2014).

[14] D. D. Sheka, V. P. Kravchuk, and Y. Gaididei, J. Phys. A 48, 125202 (2015).

[15] T. Machon and G. P. Alexander, Proc. Natl. Acad. Sci. U.S.A. 110, 14174 (2013).

[16] L. D. Landau and E. M. Lifshitz, Phys. Z. Sowjetunion 8, 153 (1935) [Ukr. J. Phys. 53, 14 (2008)].

[17] We did not consider a case of Néel domain wall here, because only walls of Bloch type are found by using full scale micromagnetic simulations.

[18] We checked it for parameters $h=5 \mathrm{~nm}, Q=1.72$.

[19] M. Heide, G. Bihlmayer, and S. Blügel, Phys. Rev. B 78, 140403 (2008).

[20] A. Hubert and R. Schäfer, Magnetic Domains: The Analysis of Magnetic Microstructures (Springer-Verlag, Berlin, 1998).

[21] The largest value of the anisotropy constant is limited by the chosen average mesh size $3 \mathrm{~nm}$ which is determined by the computational possibilities.
[22] The lower bound corresponds to a quasi-2D case when the magnetization is uniform along the normal direction, and the upper bound is determined by the condition of usage of (2).

[23] W. Scholz, MAGPAR 0.9, 2010, http://www.magpar.net/ static/magpar/doc/html/index.html.

[24] We use 12 different initial states, namely, four different states with random magnetization distributions, six states uniformly magnetized along directions $\pm \hat{\boldsymbol{x}}, \pm \hat{\boldsymbol{y}}, \pm \hat{\boldsymbol{z}}$, respectively, and two vortex states with opposite senses of the circulation. The lowest energy state obtained after applying the energy minimization procedure is considered to be the ground state for the certain set of parameters.

[25] R. P. Cowburn, D. K. Koltsov, A. O. Adeyeye, M. E. Welland, and D. M. Tricker, Phys. Rev. Lett. 83, 1042 (1999).

[26] M. Kläui, C. A. F. Vaz, L. Lopez-Diaz, and J. A. C. Bland, J. Phys. Condens. Matter 15, R985 (2003); V. P. Kravchuk, D. D. Sheka, and Y. B. Gaididei, J. Magn. Magn. Mater. 310, 116 (2007).

[27] We checked it for parameters $h=25 \mathrm{~nm}, Q=1.08$.

[28] A. Hubert, Phys. Status Solidi B 32, 519 (1969); 38, 699 (1970).

[29] P. G. De Gennes and J. Prost, The Physics of Liquid Crystals, 2nd ed. (Clarendon Press, Oxford, 1993).

[30] S. S. P. Parkin, M. Hayashi, and L. Thomas, Science 320, 190 (2008).

[31] K.-S. Ryu, L. Thomas, S.-H. Yang, and S. Parkin, Nat. Nanotechnol. 8, 527 (2013).

[32] S. Emori, U. Bauer, S.-M. Ahn, E. Martinez, and G. S. D. Beach, Nat. Mater. 12, 611 (2013).

[33] R. Streubel, J. Lee, D. Makarov, M.-Y. Im, D. Karnaushenko, L. Han, R. Schäfer, P. Fischer, S.-K. Kim, and O. G. Schmidt, Adv. Mater. 26, 316 (2014).

[34] S. Tanda, T. Tsuneta, Y. Okajima, K. Inagaki, K. Yamaya, and N. Hatakenaka, Nature (London) 417, 397 (2002).

[35] W. Beugeling, A. Quelle, and C. Morais Smith, Phys. Rev. B 89, 235112 (2014).

[36] N. A. Porter, J. C. Gartside, and C. H. Marrows, Phys. Rev. B 90, 024403 (2014).

[37] S. Nakamura, K. Wakabayashi, A. Yamashiro, and K. Harigaya, Physica E 22, 684 (2004).

[38] Z. L. Guo, Z. R. Gong, H. Dong, and C. P. Sun, Phys. Rev. B 80, 195310 (2009).

[39] A. D. Güçlü, M. Grabowski, and P. Hawrylak, Phys. Rev. B 87, 035435 (2013).

[40] J.-W. Jiang, J.-S. Wang, and B. Li, J. Appl. Phys. 108, 064307 (2010).

[41] X. Wang, X. Zheng, M. Ni, L. Zou, and Z. Zeng, Appl. Phys. Lett. 97, 123103 (2010).

[42] J. Gravesen and M. Willatzen, Phys. Rev. A 72, 032108 (2005).

[43] Z. Li and L. R. Ram-Mohan, Phys. Rev. B 85, 195438 (2012).

[44] V. M. Fomin, S. Kiravittaya, and O. G. Schmidt, Phys. Rev. B 86, 195421 (2012).

[45] K. Yakubo, Y. Avishai, and D. Cohen, Phys. Rev. B 67, 125319 (2003).

[46] D. J. Ballon and H. U. Voss, Phys. Rev. Lett. 101, 247701 (2008). 